COLO-HEP-285

\title{
An Effective Model for Crumpling in Two Dimensions?
}

\author{
C.F. Baillie \\ Physics Dept. \\ University of Colorado \\ Boulder, CO 80309 \\ USA \\ and \\ D.A. Johnston \\ Dept. of Mathematics \\ Heriot-Watt University \\ Riccarton \\ Edinburgh, EH14 4AS \\ Scotland
}

October 27, 2018

\begin{abstract}
We investigate the crumpling transition for a dynamically triangulated random surface embedded in two dimensions using an effective model in which the disordering effect of the $X$ variables on the correlations of the normals is replaced by a long-range "antiferromagnetic" term. We compare the results from a Monte Carlo simulation with those obtained for the standard action which retains the $X$ 's and discuss the nature of the phase transition.
\end{abstract}


It is now clear that the pathologies of the continuum Polyakov action for the bosonic string [1] in physical dimensions $(d>1)$

$$
Z=\int D g D X \exp \left(-\frac{1}{2} \int d^{2} x \sqrt{g} g^{a b} \partial_{a} X^{\mu} \partial_{b} X_{\mu}\right)
$$

are also manifest in the discretized Euclidean form of the action used in simulations

$$
Z=\sum_{T} \int \prod_{i} D X_{i} \exp \left(-\frac{1}{2} \sum_{<i j>}\left(X_{i}^{\mu}-X_{j}^{\mu}\right)^{2}\right) .
$$

In this simple gaussian action the continuum integration over the metric is replaced by a summation over triangulations of the discretized worldsheet, the variables $X_{i}$ which specify the embedding of the surface typically live at the nodes of the triangulation and the sum is over the edges $\langle i j\rangle$. Both microcanonical simulations, in which the number of nodes was fixed, and grand canonical simulations, in which it varied, were carried out and it was discovered that the worldsheets generated by the action were very crumpled and failed to give a non-trivial continuum limit [2].

A possible remedy, suggested by Polyakov in [3], was the inclusion of a stiffness term in the action which could be written using either of the two equivalent forms of the extrinsic curvature squared

$$
\begin{aligned}
K^{2} & =\int d^{2} x \sqrt{h}\left(\Delta_{h} X^{\mu}\right)^{2} \\
& =\int d^{2} x \sqrt{h} h^{a b} \nabla_{a} \hat{n}_{i}^{\mu} \nabla_{b} \hat{n}_{\mu}^{i} .
\end{aligned}
$$

Subsequent simulations revealed that discretizations of the first version were also afflicted by pathologies [1] but that a discretization of the second form

$$
S_{e}=\sum_{\Delta_{i}, \Delta_{j}}\left(1-\hat{n}_{\Delta_{i}} . \hat{n}_{\Delta_{j}}\right)
$$

where the $\Delta$ 's are adjacent triangles and the $\hat{n}$ 's normals, offered the possibility of defining a continuum limit at a second order phase transition where the string tension scaled [5]. Some recent simulations have cast doubt on the second order nature of the transition, but it appears that the requisite scaling of the string tension may still be taking place [6]. Other alternative actions are also being investigated [7].

The extrinsic curvature squared term in eq. 0 may also be written as

$$
S_{e}=\sum_{\Delta_{i}, \Delta_{j}}\left(1-\cos \theta_{i j}\right)
$$

where the $\theta_{i j}$ is the angle between the adjacent normals. In the degenerate case of a surface embedded in just two dimensions it is still possible to write this term using Ising-like variables $\sigma= \pm 1$

$$
S_{e}=\sum_{<i j>}\left(1-\sigma_{i} \sigma_{j}\right)
$$

as the cosine will always take the value \pm 1 . (Note that these Ising variables live on the dual of the triangulation.) This prompted Renken and Kogut to suggest that if one ignored the effect of the $X$ variables in the gaussian term one might expect an Ising like crumpling transition in this case as $S_{e}$ in eq. 6 is essentially the $2 d$ Ising model action. This transition would thus be second order and might serve as a model for the transition in more realistic dimensions. However, their numerical simulations of a gaussian plus extrinsic curvature squared action on a fixed triangulation using finite size scaling techniques revealed a first order transition [8]. It does appear that this model on a dynamical triangulation has a second order crumpling transition which is more in line with expectation, though it is only possible to work with much smaller meshes in this case [9].

As we have ignored the gaussian term it is natural to enquire what effect its inclusion might have on the analysis above. It was pointed out in [10] that if one used a proper time representation for the $X$ propagator of a gaussian surface action

$$
<X^{\mu}\left(\xi_{i}\right) X^{\nu}\left(\xi_{j}\right)>=\delta^{\mu \nu} \int_{\frac{1}{L^{2}}}^{\Lambda^{2}} \frac{d \alpha}{4 \pi \alpha} \exp \left(-\alpha\left(\xi_{i}-\xi_{j}\right)^{2}\right)
$$


where $L$ is an infrared cutoff and $\Lambda$ an ultraviolet cutoff, then the normal-normal correlations were of the form

$$
<n\left(\xi_{i}\right) \cdot n\left(\xi_{j}\right)>\simeq-\frac{A}{\left(\xi_{i}-\xi_{j}\right)^{4}}
$$

The constant $A$ depends on the dimension in which the surface is embedded 1 . From eq. 8 it is clear that the gaussian term induces an antiferromagnetic interaction between the normals. This suggests a possible interpretation of the crumpling transition as arising from the competition between the gaussian "antiferromagnetic" term and the extrinsic curvature "ferromagnetic" term, which tends to order the normals.

In this paper we test this interpretation for a surface embedded in two dimensions by comparing the behavior of the "effective" action where the gaussian term has been replaced by a term mimicing its effect on the normal spins,

$$
S=A \sum_{i j} \frac{1}{r_{i j}^{4}} \sigma_{i} \sigma_{j}+\lambda \sum_{<i j>}\left(1-\sigma_{i} \sigma_{j}\right),
$$

with the standard action we simulated in [9]

$$
S=\frac{1}{2} \sum_{<i j>}\left(X_{i}^{\mu}-X_{j}^{\mu}\right)^{2}+\lambda \sum_{<i j>}\left(1-\sigma_{i} \sigma_{j}\right)
$$

where $\lambda$ is the ferromagnetic coupling proportional to the inverse temperature. For convenience we set the constant $A=1$ in the simulations. Note that the sum in the first term of eq. 9 is over all the spins so we have a long-range interaction. Moreover $r_{i j}$ is the intrinsic distance between the spins $\sigma_{i}$ and $\sigma_{j}$, i.e. the shortest path (number of links) between points $i$ and $j$ in the triangulation. In fact since there are no $X$ variables in eq.9, the triangulation is no longer embedded in any dimension and there are no extrinsic distances. This means that we can no longer measure the radius of gyration to see how the size of the surface is changing, though we can measure the contribution of the extrinsic curvature squared term eq.6 which we would expect to be small in a smooth phase and large in a crumpled phase.

The action in eq 9 is rather similar to that suggested in [11] to explain the phenomenon of spin canting and reentrance ${ }^{2}$

$$
S=-J_{0} \sum_{<i j>} \vec{S}_{i} \cdot \vec{S}_{j}+2 \epsilon \sum_{i j} \frac{1}{r_{i j}^{d+\sigma}} \vec{S}_{i} \cdot \vec{S}_{j}
$$

where the $\vec{S}$ 's are classical two-component spins and the sum in the second term is restricted to $r_{i j}<R$, where $R$ is some cutoff. The authors in 11 were interested in $d=3$ and $\sigma<2$ and argued that the competition between the short range $J_{0}$ term and the long-range $\epsilon$ term accounted for canting. Actions of the form

$$
S=-J_{0} \sum_{i j} \frac{1}{r_{i j}^{\alpha}} \sigma_{i} \sigma_{j}
$$

have also been considered [12], and display phase transitions for $d<\alpha<2 d$. In our case the $1 / r^{4}$ interaction induced between all the normals is apparently on the borderline $\alpha=2 d$ if we assume $d=2$, but direct measurement of $d$ on the highly irregular meshes generated by $2 \mathrm{~d}$ quantum gravity that we use gives $d>2$. However our interaction is antiferromagnetic, so it appears that the analogy with the canting action is closer as we have two competing terms, one "ferromagnetic" and one "antiferromagnetic", and observe a transition as we vary their relative strengths.

The most difficult part in performing a Monte Carlo simulation of the action in eq.9 is calculating the intrinsic distance $r_{i j}$ (represented as a matrix) between all points $i, j$ on the random triangulation. There is a straight-forward algorithm which does this but it is $O\left(N^{2}\right)$, where $N$ is the number of points in the triangulation. This makes it prohibitively expensive to simulate dynamical random triangulations, since after every change to the triangulation one would have to recalculate $r_{i j}$ (even though the changes to the triangulation would consist only of the standard "flip" move which is local this would still produce non-local changes in the intrinsic distance matrix $r_{i j}$ ). Therefore all our simulations use fixed random

\footnotetext{
${ }^{1}$ The considerations of $[10$ were for rigid or crystalline surfaces but they are essentially unchanged for dynamical surfaces.

${ }^{2}$ Reentrance is the behavior displayed by metallic spin glasses such as AuFe which have a paramagnetic to ferromagnetic transition at some $T_{c}$ and a ferromagnetic to spin-glass transition at some $T_{f}<T_{c}$. Below $T_{f}$ spins tend to rotate away from an external field, which is termed canting.
} 
triangulations so that we have only to calculate $r_{i j}$ once at the beginning. As our model is essentially an Ising model coupled to two-dimensional quantum gravity, albeit with an extra long-range term, we have chosen to use random triangulations coming from pure two-dimensional quantum gravity simulations as was done in 13 .

We have performed simulations on three sizes of random triangulation of spherical topology with total number of points $N=100,500$ and 1000. For each $N$ we ran at roughly 20 values of $\lambda$ between 1 and 3. After thermalizing we do 100000 Monte Carlo updates using the standard Metropolis algorithm, measuring the energy, magnetization and spin-spin correlation function after every update which allows us to calculate autocorrelation times and correlation length. We find, as expected for the local Metropolis algorithm, that the autocorrelation time $\tau$ scales as the square of the correlation length $\xi$; in fact for the energy, by fitting $\tau \sim \xi^{z}$, we extract a dynamical critical exponent $z=2.1(2)$. From the fluctuations in the energy and magnetization we obtain the specific heat and susceptibility in the usual manner.

In Fig. 1 we show the total energy $E$ from the largest system simulated $N=1000$ (graphs from $N=100$ and 500 look almost identical) and its two parts $-E_{l r}$ is the antiferromagnetic long-range part (first term in eq.9 with $\mathrm{A}=1$ ) and $E_{n n}$ is the ferromagnetic nearest-neighbor part (second term in eq.9 including $\lambda$ ). In Fig. 2 we show the total magnetization $M$, from all three simulations. At small $\lambda$, which corresponds to high temperature, we see from Fig. 1 that most of the energy is in the ferromagnetic piece $E_{n n}$ since the spins are disordered, which we would expect for a "crumpled" phase $-M$ is small in Fig. 2. Conversely, at large $\lambda$ (low temperature) the spins line up so $M \rightarrow 1$ and most of the energy resides in the antiferromagnetic $E_{l r}$. In this smooth phase the contribution from the extrinsic curvature squared $E_{n n}$ is small. From these we deduce there is some sort of crumpling transition at around $\lambda=2.3-2.4$. Also from Fig. 2 we see that as the system size increases the "jump" in $M$ becomes more abrupt, perhaps signaling the appearance of a first order phase transition. However there is no corresponding jump in the total energy so this seems unlikely.

Stronger evidence of the phase transition not being of first order comes from the specific heat, shown in Fig. 3. For a first order phase transition, standard finite-size scaling theory predicts that for a two-dimensional system of size $N=L \times L$, the specific heat peak

$$
C_{\max }=A L^{2}+B
$$

whereas for a second order transition

$$
C_{\max }=A^{\prime} L^{\alpha / \nu}+B^{\prime}
$$

We do not know a priori that our system is two-dimensional so we must write $L=N^{\frac{1}{d}}$, where $d$ is the fractal dimension of the random triangulation. In fact, numerical simulations for pure $2 \mathrm{~d}$ quantum gravity [14] and for $2 \mathrm{~d}$ quantum gravity coupled to Potts models 15$]$ yield $d \approx 2.7$ or 2.8 ; and an analytical calculation predicts that $d$ lies between 2 and 3 for pure 2 d quantum gravity [16]. Thus we fit the specific heat peak to

$$
C_{\max }=A^{\prime} N^{\alpha / \nu d}+B^{\prime}
$$

for our three values of $N$ obtaining $\alpha / \nu d=0.12(2)$. Thus a first order phase transition is apparently ruled out. One could argue that our systems are too small to see the true scaling of $C_{\max }$, however Renken and Kogut managed to see its first order scaling in their model on systems of size 576 and 1024 [8]. It should be noted, however, that they carried out their simulations on a regular, fixed lattice rather than the highly disordered lattice we are using, so the different behavior we observe might be due to the lattice rather than the absence of the gaussian term.

If we assume that the phase transition is second order and use the scaling relation

$$
\nu d=2-\alpha
$$

we obtain $\alpha=0.20(3)$ and $\nu d=1.8(3)$. Now, from our measurements of the correlation length, we can obtain $\nu$ by fitting

$$
\xi \sim\left|\lambda_{c}-\lambda\right|^{-\nu}
$$

for $\lambda<\lambda_{c}$, with $\lambda_{c}=2.35$. This value for $\lambda_{c}$ is obtained from the peak in the susceptibility, shown in Fig. 4. This leads to $\nu=0.7(1)$. Therefore we estimate $d=2.6(8)$. Unfortunately the error here is rather large but nevertheless the numbers are consistent. As a final check we could assume $\alpha=0$ (as is 
the case for the phase transition in the usual $2 d$ Ising model), then $\nu d=2$ so we eliminate one source of error and obtain $d=2.9(4)$.

At this point we could tentatively conclude that our data is consistent with the phase transition being second order. However, it is interesting to note similarities with data from numerical simulations of (gaussian) spin glasses which typically have a rather broad peak in the specific heat and a susceptibility peak at lower temperature 17] (which corresponds to higher $\lambda$ ). If we look at Fig.3 and Fig.4 we see just this behavior. The very weak growth of the peak in the specific heat that we have measured could be a finite size effect masking a negative $\alpha$ (in the Sherrington/Kirkpatrick spin glass model $\alpha=-1$ [17]) 3. Moreover, at the phase transition in our model we have competing interactions which typically leads to spin-glass type behavior. As these interactions are still present in the original model with gaussian plus extrinsic curvature-squared terms this suggests that the low $\lambda$, crumpled phase in this model could conceivably be spin-glass like rather than paramagnetic. A simulation by Heermann [18] has, in fact, found that the radius of gyration $X 2$ is non self-averaging in the crumpled phase, a behavior found in some observables in spin glasses.

To summarize the numerical results and speculations: we have simulated an effective model for crumpling in two dimensions in which the $X$ variables are replaced by an antiferromagnetic term that mimics their effect on the normals. We have found what appears to be a weak second (or possibly higher) order transition, rather than the first order transition of [8], which retained the $X \mathrm{~s}$. We have pointed out that this might be due to the differing underlying fixed meshes in the simulations rather than the absence of the $X$ variables. We have also speculated that the competing nature of the interactions at the transition may give a spin-glass like phase at low $\lambda$ rather than a paramagnetic phase and presented some numerical support for this view.

It would be interesting to repeat the simulation on a fixed regular mesh to compare directly with [8] and to extend the effective model work to the more realistic case of a surface embedded in three dimensions, where one would employ continuous spins. The nature of the low $\lambda$ ("crumpled") phase also merits further investigation for both the effective model and the original action.

This work was supported in part by NATO collaborative research grant CRG910091. CFB is supported by DOE under contract DE-AC02-86ER40253 and by AFOSR Grant AFOSR-89-0422.

\footnotetext{
${ }^{3}$ Recent very high statistics simulations of a gaussian plus extrinsic curvature-squared action in three dimensions [6] have also found very little, if any, growth in the peak.
} 


\section{References}

[1] A. M. Polyakov, Phys. Lett. 103B (1981) 207.

[2] J. Ambjorn, B. Durhuus and J. Fröhlich, Nucl. Phys. B257 (1985) 433;

D. Boulatov, V. Kazakov, I. Kostov and A. Migdal, Nucl. Phys. B275 (1986) 241;

A. Billoire and F. David, Nucl. Phys. B275 (1986) 617;

J. Jurkiewicz, A. Krzywicki and B. Petersson, Phys. Lett. 168B (1986) 273;

B. Durhuus, J. Fröhlich and T. Jonsson, Nucl. Phys. B240 (1984) 453;

J. Ambjorn, B. Durhuus, J. Fröhlich and P. Orland, Nucl. Phys. B270 (1986) 457;

B. Durhuus, J. Fröhlich and T. Jonsson, Nucl. Phys. B225 (1983) 185.

[3] A. Polyakov, Nucl. Phys. B268 (1986) 406;

H. Kleinert, Phys. Lett. 174B (1986) 335.

[4] M. Baig, D. Espriu and J. Wheater, Nucl. Phys. B314 (1989) 609;

R. Harnish and J. Wheater, Nucl. Phys. 350 (1991) 861.

[5] S. Catterall, Phys. Lett. 220B (1989) 332;

C. F. Baillie, D. A. Johnston and R. D. Williams, Nucl. Phys. B335 (1990) 469;

R. Renken and J. Kogut, Nucl. Phys. B354 (1991) 328;

C. F. Baillie, R. D. Williams, S. M. Catterall and D. A. Johnston, Nucl. Phys. B348 (1991) 543.

[6] J. Ambjorn, J. Jurkiewicz, S. Varsted and A. Irback, Phys. Lett. B275 (1992) 295;

A. Irback, J. Jurkiewicz and S. Varsted, "Measuring the string tension in random surface models with extrinsic curvature", NBI-HE-91-32 preprint (Aug. 1991).

[7] C.F. Baillie and D.A. Johnston, Phys. Rev. D45 (1992) ????.

[8] R. Renken and J. Kogut, Nucl. Phys. B350 (1991) 554.

[9] C. F. Baillie and D. A. Johnston, Phys. Lett. 258B (1991) 346.

[10] J. Ambjørn, B. Durhuus and T. Jonsson, Nucl. Phys. B316 (1989) 526.

[11] A. vanEnter and J. vanHemmen, Phys. Rev. B31 (1985) 603.

[12] O. Penrose, J. Stat. Phys. 45 (1986) 69.

[13] M. E. Agishtein and C. F. Baillie, Mod. Phys. Lett. A6 (1991) 1615.

[14] M.E. Agishtein and A.A. Migdal, Mod. Phys. Lett. A7 (1992) 1039.

[15] C. F. Baillie and D. A. Johnston, "A Numerical Test of KPZ Scaling: Potts Models Coupled to Two-Dimensional Quantum Gravity", to appear in Mod. Phys. Lett. A (1992);

C. F. Baillie and D. A. Johnston, "Multiple Potts Models Coupled to Two-Dimensional Quantum Gravity", to appear in Phys. Lett. B (1992).

[16] H. Kawai and M. Ninomiya, Nucl. Phys. B336 (1990) 115.

[17] K. Binder and A. Young, Rev. Mod. Phys. 58 (1986) 801.

[18] D.W. Heermann, Int. J. Mod. Phys. C2 (1991) 613.

\section{Figure Captions}

Fig. 1. Energy for the $\mathrm{N}=1000$ simulation.

Fig. 2. Magnetization for all three $(\mathrm{N}=100,500$ and 1000) simulations.

Fig. 3. Specific heat for all three simulations.

Fig. 4. Susceptibility for all three simulations. 